\title{
U ne approche des coûts de production des semences fourragères dans la zone soudano-sahélienne du Cameroun
}

\author{
J. Onana ${ }^{1}$ S. Yonkeu ${ }^{2}$
}

\section{Mots-clés}

Légumineuse fourragère - Production de semences - Zone soudano-sahélienne - Cameroun.

\begin{abstract}
Résumé
Les coûts de production des semences de sept légumineuses fourragères ont été évalués en zone soudano-sahélienne du Cameroun. Ils étaient inférieurs à $1000 \mathrm{FCFA} / \mathrm{kg}$ pour Calopogonium mucunoides, Cajanus cajan, Desmodium distortum, Mucuna pruriens et Stylosanthes hamata (1 FCFA $=0,01 \mathrm{FF}$ ). La production de semences de Centrosema pascuorum et de Macroptilium lathyroides, avec des coûts respectifs de 1890 et de $3025 \mathrm{FCFA} / \mathrm{kg}$, ne semble pas adaptée à la vulgarisation dans la zone de l'étude.
\end{abstract}

\section{INTRO DUCTION}

Dans la province du Nord Cameroun, les pâturages naturels sont essentiellement composés de graminées et constituent encore la principale source de fourrage pour les herbivores domestiques. Leur valeur alimentaire varie cependant d'une région à l'autre en fonction de la composition floristique. Pendant la saison sèche, cette valeur alimentaire est presque nulle et les animaux ont alors recours à d'autres sources fourragères parmi lesquelles les légumineuses qui en général sèchent plus tardivement et conservent à l'état sec une valeur nutritive supérieure à celles des graminées. Elles peuvent d'ailleurs être stockées pour la saison sèche. Cette famille botanique est relativement peu abondante dans les pâturages de la zone de l'étude et les espèces spontanées sont peu productives d'où l'intérêt d'introduire les espèces exotiques. Des espèces herbacées ou ligneuses ont ainsi été introduites depuis 1984. En dehors de cet intérêt fourrager, certaines des légumineuses introduites à la Station de recherches zootechniques et vétérinaires de Garoua $(1,3,5)$ sont utilisées à d'autres fins. Par exemple, Mucuna pruriens, à Sanguéré-Bamé, sert à lutter contre les adventices dans les vergers. Les graines de Mucuna pruriens sont torréfiées et consommées comme stimulant au même titre que le café dans de nombreuses localités (Mowo, Djalingo, Bamé). Les organismes de développement utilisent également ces plantes comme plantes de couverture afin de lutter contre l'érosion des sols et améliorer leur fertilité.

L'un des freins à une vulgarisation accrue des espèces fourragères prometteuses est le coût de production des semences qui demeure

1. Institut de recherches agricoles pour le développement, Station polyvalente, BP 1073, Garoua, Cameroun

Tél. : +237272084; fax : +237272333 ou 2337 ou 2898

2. EIER, 03 BP 7023, Ouagadougou 03, Burkina-Faso élevé pour le paysan. Ces travaux ont été menés pour déterminer, dans des conditions proches de celles des exploitations paysannes, le coût de production des semences d'espèces qui intéressent le plus les paysans et les organismes de développement.

\section{MATERIEL ET METHODES}

\section{Milieu de l'étude}

L'étude a été menée en milieu paysan à Ouro-Labo II et à SanguéréPaul, deux villages situés près de la ville de Garoua, capitale de la province du Nord Cameroun. La zone géographique englobant ces deux sites est comprise entre $8^{\circ} 30^{\prime}$ et $9^{\circ}$ de latitude $\mathrm{N}$ et entre $13^{\circ}$ et $14^{\circ} 05^{\prime}$ de longitude E. Le climat est de type soudano-sahélien avec deux saisons de durée égale : une saison sèche de novembre à avril et une saison des pluies de mai à octobre. Les précipitations moyennes de 1990 à 1995 ont été de $950 \mathrm{~mm}$. Les températures mensuelles moyennes ont varié entre 27 et $30^{\circ} \mathrm{C}$. Le mois le plus chaud a été avril $\left(32{ }^{\circ} \mathrm{C}\right)$. Les principales activités économiques de la région sont l'élevage et l'agriculture. Malgré la diversité pédologique observée dans la région, les essais ont été menés sur sols ferrugineux, dans d'anciennes jachères arbustives à Combretum collinum. Ces sols couvrent la majeure partie du secteur phytogéographique soudano-sahélien au Cameroun.

\section{Matériel végétal}

Les espèces de l'étude étaient en observation en station depuis plusieurs années. La première partie des travaux a porté sur l'étude du comportement : adaptation au milieu, production potentielle de biomasse et leur utilisation par les animaux $(1,3,4,5)$. Elles se ressèment toutes naturellement chaque année. Le degré de 
couverture initial est cependant variable suivant les espèces. Il est de l'ordre de 80 p. 100 pour Calopogonium mucunoides, Stylosanthes hamata et Mucuna pruriens; de 40 à 60 p. 100 pour Desmodium distortum et Macroptilium lathyroides ; en général faible pour Centrosema pascuorum et Cajanus cajan. En outre quatre espèces ont une pérennité variant entre deux et quatre ans : Cajanus cajan, Desmodium distortum, Macroptilium lathyroides et Stylosanthes hamata alors que les quatre autres espèces se comportent en annuelles.

Des observations sur la phénologie des espèces ont été réalisées en complément aux études de comportement déjà disponibles (5). Ces observations ont porté sur :

- le mode de floraison (groupée ou étalée dans le temps) ;

- le changement de coloration des gousses en fonction de leur stade de développement.

\section{Mise en place des espèces et collecte des données}

L'essai a été mis en place en juillet 1994 chez cinq particuliers dont trois à Sanguéré-Paul et deux à Ouro-Labo II. La production de semences a été évaluée en fin de campagne (octobre 1994 à avril 1995 selon les espèces). Chaque paysan disposait d'une surface minimale de deux hectares sur laquelle a été installée chacune des huit accessions étudiées. La mise en place des parcelles expérimentales avait été précédée au cours de la campagne agricole 1993-1994 d'une phase de prévulgarisation afin de montrer aux paysans l'intérêt des plantes utilisées et de les initier aux techniques de leur installation.

Les fiches techniques de vulgarisation sur lesquelles étaient mentionnés les itinéraires techniques de mise en place, d'entretien, les périodes d'apparition des différentes phases du cycle biologique ainsi que les techniques de récolte des graines des diverses espèces ont été remises aux paysans concernés par l'essai.

Le sol a été labouré avec des bœufs de trait. La mise en place des espèces a été faite selon deux modalités de semis au cours de la première semaine de juin, dès que les pluies étaient déjà bien installées :

- le semis en lignes espacées de $80 \mathrm{~cm}$ à des densités de $5 \mathrm{~kg} / \mathrm{ha}$ de semences pour Calopogonium mucunoides, Centrosema pascuorum, Desmodium distortum, Stylosanthes hamata et Macroptilium lathyroides;

- le semis en poquets de deux graines espacés de 100 x $100 \mathrm{~cm}$ pour Cajanus cajan (15 kg de semences/ha) et Mucuna pruriens (18 kg de semences/ha).

Deux sarclages ont été effectués pour entretenir les plantations après les semis. Dans la province, les coûts de sarclage étaient généralement négociables et obéissaient à la loi de l'offre et de la demande. Ils avoisinaient cependant au cours d'une saison agricole autour d'une moyenne qui, en 1995, était de 3500 FCFA pour un quart d'hectare. Chaque sarclage nécessitait six journées de travail humain (jt) pour un quart d'hectare $(1 \mathrm{jt}=$ une journée de 8 heures consacrées à un travail agricole par un homme adulte et valide ou un actif agricole).

Les sols étant généralement déficients en phosphore, cet élément a été apporté quatre semaines après la levée sous forme de superphosphate triple à raison de quatre sacs de $50 \mathrm{~kg} / \mathrm{ha}$ (la forme commercialisée sur le marché local était conditionnée à 46 p. 100 de surperphosphate par unité de poids d'engrais).
Comme il s'agissait d'une production nouvelle non directement destinée à l'alimentation humaine, la démarche expérimentale énoncée ci-dessus visait principalement à se rapprocher des conditions culturales du paysan moyen.

Des visites hebdomadaires sur le terrain permettaient de vérifier que les calendriers d'exécution des travaux étaient réalisés suivant les prescriptions des fiches techniques.

La quantité de semences produites sur toute la surface réelle d'un quart d'hectare en observation a été collectée manuellement puis pesée pour chaque espèce. Cette section du travail a nécessité $50 \mathrm{jt}$ pour Cajanus cajan, 96 jt pour Calopogonium mucunoides, $80 \mathrm{jt}$ pour Centrosema pascuorum, 50 jt pour Desmodium distortum, 72 jt pour Macroptilium lathyroides, 20 jt pour Mucuna pruriens et 72 jt pour Stylosanthes hamata.

\section{Calcul du prix de revient des semences}

Le prix de revient $(\mathrm{Pv})$ des semences de chaque accession a été évalué en francs de la Communauté financière africaine par kilogramme produit à partir de la moyenne des productions des cinq parcelles expérimentales. Il comprenait :

- les frais communs (Fc) (en FCFA) qui étaient invariables quelle que soit la culture. Ils portaient sur la préparation du terrain (dessouchage, labour), le semis, le sarclage, et l'achat et l'épandage des engrais ;

- les frais spécifiques à chaque accession (Fs) (en FCFA) qui étaient variables et dépendaient étroitement de la production semencière de chaque accession. Ils comprenaient :

- les frais des produits phytosanitaires au moment de la commercialisation ; 400 FCFA pour $40 \mathrm{~g}$ de Marshal 25 ST (un carbosulfan qui se présente sous forme de poudre sèche de couleur rouge), nécessaire au traitement de $50 \mathrm{~kg}$ de semences ;

- les frais d'emballage qui dépendaient aussi du type de conditionnement sollicité par l'acheteur. Ils étaient de 40 FCFA pour un sac en plastique de 1 à $10 \mathrm{~kg}$ de semences, de 150 FCFA pour un sac en jute de $50 \mathrm{~kg}$ et de 300 FCFA pour un sac de $100 \mathrm{~kg}$;

- les frais de main d'œuvre pour le conditionnement et la mise en sacs de semences ; 750 FCFA par sac de $100 \mathrm{~kg}$, soit 7,5 FCFA/kg ;

- les prix d'achat des semences de pré-base auprès des instituts de recherche et de leur récolte dans les parcelles de multiplication étaient, contrairement aux trois points précédents, étroitement liés à la taille des graines et au potentiel de production de chaque espèce.

Le prix de revient des semences était donc :

$$
\mathrm{Pv}(\mathrm{FCFA} / \mathrm{kg})=\frac{(\mathrm{Fc}+\mathrm{Fs}) / \mathrm{ha}}{\mathrm{P}(\mathrm{kg} / \mathrm{ha})}
$$

\section{Test de viabilité des semences}

La qualité des semences produites a été évaluée par des tests classiques de germination. Cent graines ont été utilisées à cet effet pour chaque espèce. Pour les grosses graines (Mucuna pruriens), des petits bacs de germination remplis de sciure fine de menuiserie ont été utilisés ; pour les autres espèces à petites graines, des boîtes de Pétri avec des fonds recouverts de papier buvard ont été employés. Un arrosage journalier a été effectué pour entretenir l'humidité des semences. 


\section{RESU LTATS}

\section{Phénologie des espèces}

Les cycles phénologiques de la reproduction des espèces étudiées tels qu'ils ont été observés pendant trois ans d'études préliminaires en station et au cours des présents travaux sont résumés dans le tableau I. Ces résultats se rapportent aux semis effectués dans l'année.

Avec des resemis naturels des espèces étudiées, comme Calopogonium mucunoides, Stylosanthes hamata, ainsi qu'avec les rejets des espèces pérennes, comme Macroptilium lathyroides, qui ont souvent eu lieu dès l'arrivée des pluies parasites de la fin de la saison sèche, la phase de reproduction a débuté plus précocement après l'installation effective de la saison des pluies.

Plusieurs signes permettent de constater visuellement la maturation des fruits des différentes espèces :

- le noircissement des gousses chez Mucuna pruriens et Macroptilium lathyroides ;

- le blanchissement des gousses chez Centrosema pascuorum;

- le jaunissement des gousses chez Calopogonium mucunoides.

On observe deux types de reproduction sexuée chez les espèces étudiées :

- la reproduction étalée. La floraison débute 11 à 12 semaines après la germination des graines et se poursuit pendant tout le reste de la saison des pluies. Elle se prolonge quelques semaines encore pendant la saison sèche alors que la récolte des fruits matures issus des premières fleurs a déjà commencé. C'est le cas chez Centrosema pascuorum et Macroptilium lathyroides. Si la première espèce n'est pas autochore, il n'en est pas de même pour la deuxième dont les gousses arrivées à maturité explosent et dispersent abondamment les graines. La récolte des gousses est faite tous les deux jours. La période de récolte est alors allongée entrainant une augmentation des frais relatifs à la récolte et donc des coûts de production des semences ;

- la reproduction groupée. La floraison débute 12 à 13 semaines après la germination, période pendant laquelle plus de 80 p. 100 des inflorescences sont formées. C'est le mode de reproduction chez Mucuna pruriens et Calopogonium mucunoides. Il permet de mieux organiser la récolte des semences sur un laps de temps assez court.

\section{Production et prix de revient des semences}

Les frais communs calculés au cours de l'essai pour la préparation d'un hectare ont été :

- 33000 FCFA pour le dessouchage ;

- 18000 FCFA pour le labour ;

- 2000 FCFA pour le semis ;

- 28000 FCFA pour deux sarclages ;

- 42000 FCFA pour l'achat des engrais (10 500 FCFA/sac $\mathrm{x} 4$ sacs) ;

- 6000 FCFA pour l'épandage des engrais.

Le total des frais communs a été de 129000 FCFA par hectare.

Les frais spécifiques sont rapportés dans le tableau II. Les prix de revient des semences conditionnées et prêtes à la vente des différentes accessions étudiées sont présentés dans le tableau III.

Il était important de noter que, dès la deuxième campagne de production semencière, les prix de revient étaient susceptibles d'être revus à la baisse à cause de la disparition de certains postes de dépense comme le dessouchage. Par ailleurs, pour les espèces qui se ressèment naturellement, comme Calopogonium mucunoides, Stylosanthes hamata, il n'était plus nécessaire d'acheter des semences, diminuant ainsi le prix de revient.

Les productions semencières obtenues dans la présente étude sont en accord avec les moyennes signalées dans la littérature (6), même si certains résultats sont loin des productions maximales annoncées. La qualité des semences déterminée par leur pouvoir germinatif montre que celles-ci répondent bien aux normes internationales de commercialisation (80-90 p. 100 de germination).

\section{DISCUSSION ET CONCLUSION}

Toutes les espèces concernées par ce travail sont en cours de vulgarisation à grande échelle dans la zone de l'étude pour leur usage multiple (fourrage, plante de couverture, plante nettoyante, alimentaire). En 1995, $350 \mathrm{~kg}$ de Calopogonium mucunoides, $75 \mathrm{~kg}$ de Stylosanthes hamata, $90 \mathrm{~kg}$ de Cajanus cajan et $1700 \mathrm{~kg}$ de Mucuna pruriens ont été vendus à l'Ondr au Tchad et à la Sodecoton au Cameroun par le Gic-Prosan (Groupes d'initiatives communes des Producteurs des semences agropastorales du Nord)

Tableau I

Phénologie habituelle de quelques légumineuses fourragères au Nord Cameroun

\begin{tabular}{lccc} 
Espèces & & Age (en semaines) & \\
\cline { 2 - 4 } & A la floraison & A la fructification & A la récolte des semences \\
Cajanus cajan & 11 & $13-14$ & $26-28$ \\
Calopogonium mucunoides & $12-13$ & $14-15$ & 23 \\
Centrosema pascuorum & 12 & $15-16$ & $24-26$ \\
Desmodium distortum & $11-12$ & 15 & $21-23$ \\
Macroptilium lathyroides & 11 & $14-21$ & $18-28$ \\
Mucuna pruriens var. utilis & 12 & 15 & 23 \\
Mucuna pruriens var. cochinchinensis & 12 & 15 & 23 \\
Stylosanthes hamata & $12-14$ & $15-16$ & 28
\end{tabular}


Tableau II

Frais spécifiques de la production de semences de quelques espèces fourragères en zone soudano-sahélienne du Cameroun

\begin{tabular}{|c|c|c|c|c|c|c|c|}
\hline \multirow[t]{2}{*}{ Espèce } & \multirow[t]{2}{*}{$\begin{array}{c}\text { Récolte } \\
\text { des semences } \\
\text { (FCFA/ha) }\end{array}$} & \multirow[t]{2}{*}{$\begin{array}{l}\text { Traitement } \\
\text { phytosanitaire } \\
\text { (FCFA/ha) }\end{array}$} & \multirow{2}{*}{$\begin{array}{l}\text { Emballage } \\
\text { et mise en sacs } \\
\text { (FCFA/ha) }\end{array}$} & \multicolumn{3}{|c|}{$\begin{array}{c}\text { Achat de semences } \\
\text { (Campagne agricole } \\
\text { 1994/1995) }\end{array}$} & \multirow[t]{2}{*}{$\begin{array}{l}\text { Total des } \\
\text { coûts spécifiques } \\
\text { (FCFA/ha) }\end{array}$} \\
\hline & & & & $\begin{array}{c}\text { Q uantité } \\
\text { (kg/ha) }\end{array}$ & $\begin{array}{l}\text { Prix du kg } \\
\text { (FCFA) }\end{array}$ & $\begin{array}{l}\text { Coût total } \\
\text { (FCFA/ha) }\end{array}$ & \\
\hline Cajanus cajan & 140000 & 12400 & 2400 & 15 & 1500 & 22500 & 177300 \\
\hline Calopogonium mucunoides & 300000 & 9300 & 1800 & 5 & 5000 & 25000 & 336100 \\
\hline Centrosema pascuorum & 140000 & 2480 & 480 & 5 & 7500 & 37500 & 180460 \\
\hline Desmodium distortum & 140000 & 6200 & 1200 & 5 & 6000 & 30000 & 177400 \\
\hline Macroptilium lathyroides & 140000 & 1500 & 300 & 4 & 7500 & 37500 & 179300 \\
\hline $\begin{array}{l}\text { Mucuna pruriens var. utilis } \\
\text { Mucuna pruriens }\end{array}$ & 140000 & 21700 & 4200 & 18 & 3000 & 54000 & 219900 \\
\hline var. cochinchinensis & 140000 & 13950 & 2700 & 18 & 3000 & 54000 & 210650 \\
\hline Stylosanthes hamata & 200000 & 6200 & 1200 & 5 & 5000 & 25000 & 232400 \\
\hline
\end{tabular}

\section{Tableau III}

Prix de revient des semences de quelques espèces fourragères en zone soudano-sahélienne du Cameroun

\begin{tabular}{lcccr} 
Espèce & $\begin{array}{c}\text { Production } \\
\text { (P en kg/ha) }\end{array}$ & $\begin{array}{c}\text { \% de germination } \\
\text { (laboratoire) }\end{array}$ & $\begin{array}{c}\text { Total des } \\
\text { coûts spécifiques } \\
\text { (Fs en FCFA/ha) }\end{array}$ & $\begin{array}{r}\text { Prix de revient } \\
\text { des semences } \\
\text { (Pv en FCFA/kg)* }\end{array}$ \\
\hline Cajanus cajan & $800 \pm 160$ & $>75$ & 177300 & 383 \\
Calopogonium mucunoides & $600 \pm 65$ & $>90$ & 336100 & 775 \\
Centrosema pascuorum & $160 \pm 10$ & $>80$ & 180460 & 1933 \\
Desmodium distortum & $400 \pm 35$ & $>80$ & 177400 & 766 \\
Macroptilium lathyroides & $100 \pm 17$ & $>80$ & 179300 & 3083 \\
Mucuna pruriens var. utilis & $1400 \pm 120$ & $>90$ & 219900 & 249 \\
Mucuna pruriens var. cochinchinensis & $900 \pm 90$ & $>80$ & 210650 & 378 \\
Stylosanthes hamata & $400 \pm 80$ & $>80$ & 232400 & 903
\end{tabular}

* Pv $($ FCFA/kg $)=\left[(129000\right.$ FCFA + Fs)/ha $] \times[\mathrm{P}(\mathrm{kg} / \mathrm{ha})]^{-1}$

et par l'ex-station Irzv de Garoua. La contribution de l'ex-station Irzv dans ce marché a été respectivement de 15,40, 35,66, 93,88 et 32,50 p. 100 pour chacune de ces quatre espèces.

Les résultats obtenus au cours de cette étude montrent que l'activité de production de semences fourragères peut être rentable. On peut penser qu'au cours des prochaines années, le marché local pourrait être saturé si la production de semences était fortement vulgarisée en milieu paysan.

Parmi les espèces étudiées, les semences de Mucuna pruriens peuvent avoir d'autres débouchés. En effet, celles-ci sont très appréciées par les porcins (6) et les chèvres. La consommation des graines non traitées entraîne cependant des diarrhées et des vomissements sévères chez les porcins (2). Elles sont occasionnellement consommées par les paysans, mais, malgré les informations diffusées dans la littérature (7), la présence d'une substances antinutritionnelle, la L-dopa, dans les graines implique qu'il est indispensable de prendre des précautions lors de son utilisation. Une synthèse récente des travaux réalisés sur la toxicité de ces graines signale que cette substance est éliminée soit en faisant bouillir les graines pendant une à deux heures tout en remplaçant plusieurs fois l'eau de cuisson, soit en les torréfiant à feu doux. Les graines ainsi traitées sont sans danger pour l'homme et les porcins (2).
Des mesures réglementaires visant à promouvoir l'essor du monde rural à travers les activités agropastorales ont été mises en place par les pouvoirs publics. C'est le cas de la loi n ${ }^{\circ} 992 / 006 \mathrm{du}$ 14 août 1992 et de son décret d'application $n^{\circ}$ 92/455/PM du 23 novembre 1992 régissant la constitution des Gic. Ces dispositions réglementaires présentent l'avantage de favoriser la commercialisation hors taxes des productions par les organisations paysannes. C'est ainsi que fut créé le Gic-Prosan le 21 novembre 1996 à Garoua. Ce groupe qui est actuellement le seul légalement en activité dans le domaine des semences fourragères conditionne, puis commercialise les produits de ses membres. Les bénéfices sont répartis proportionnellement à l'investissement financier de chaque membre conformément aux statuts internes du Gic. Chaque groupe adoptant librement ses statuts, d'autres modes d'organisation de la commercialisation et de la production peuvent être définis.

Ces initiatives au niveau de la politique de libéralisation du secteur semencier, associées à la diversité agro-écologique du Cameroun, concourent actuellement à en faire un moteur potentiel en matière de production de semences pour la sous-région d'Afrique centrale.

Grâce au ministère de l'Agriculture (Minagri) et à celui de la Recherche scientifique et technique (Minrest), à travers le 
Programme national de vulgarisation et de recherches agricoles (Pnvra), la diffusion à large échelle de ces légumineuses à usages multiples a débuté en 1998 .

Les espèces dont les coûts de production des semences étaient inférieurs à $1000 \mathrm{FCFA} / \mathrm{kg}$ pouvaient être considérées comme aptes à la vulgarisation dans la zone écologique si le paysan y trouvait son intérêt. En effet, plusieurs semences alimentaires comme celles du niébé avaient un prix de vente de près de 1000 FCFA/kg qui ne décourageait pas les producteurs de les acheter. Des coûts de production plus élevés auraient été hors de portée des éleveurs moyens. Les autres espèces devraient faire l'objet d'essais de nouvelles techniques de multiplication afin d'aboutir à une baisse des coûts de production et de les rendre plus accessibles aux utilisateurs.

\section{BIBLIO GRAPHIE}

1. KLEIN H.D., 1994. Introduction des légumineuses dans la rotation céréale-cotonnier au Nord Cameroun : gestion et utilisation. MaisonsAlfort, France, Cirad-emvt, 184 p. + annexes.

\section{Summary}

O nana J., Yonkeu S. Forage seed production in the SudanoSahelian zone of Cameroon

Seed production costs of seven forage legumes were evaluated in the Sudano-Sahelian region of Cameroon. They were under $1000 \mathrm{FCFA} / \mathrm{kg}$ for Calopogonium mucunoides, Cajanus cajan, Desmodium distortum, Mucuna pruriens and Stylosanthes hamata. Production costs of 1890 and 3025 FCFA $/ \mathrm{kg}$ for Centrosema pascuorum and Macroptilium lathyroides, respectively, did not appear attractive for the study area.

Key words: Feed legume - Seed production - Sudano-Sahelian region - Cameroon.
2. LORENZETTI F, MACLSAAC S, ARNASON J.T., AWANG D.V.C. BUKLES D., 1998. The phytochemistry, toxicology, and food potential of Velvetbean (M ucuna adans. spp., Fabaceae). In : Buckles D., Etéka A., O sina O., Galiba M., Galiano G. Eds, Plantes de couverture en Afrique de l'O uest: Une contribution à l'agriculture durable. Ibadan, Nigeria, lita, p. 67-84

3. ONANA J., 1994. Q uatre années d'essais sur les espèces fourragères exotiques en zone soudanienne du Cameroun (1984-1987). Rapport technique $n^{\circ}$ 2. Garoua, Cameroun, Srzv/section Agrostologie, 5 p. + annexes.

4. ONANA J., 1994. Les ressources fourragères du Nord Cameroun : diversité, gestion et conservation. In : Acte du colloque national sur la conservation et I'utilisation des ressources phytogénétiques, Yaoundé, Cameroun, 23-25 mars 1994. Yaoundé, Cameroun, Minrest, p. 166-180.

5. ONANA J., YONKEU S., 1994. Adaptation au milieu de quelques espèces exotiques introduites à Garoua entre 1984 et 1987. Revue sci. Dév., 3 : $71-78$

6. PU GLIESE P.L., 1984. Les graines de légumineuses d'origine tropicale en alimentation animale. Maisons-Alfort, France, lemvt, $186 \mathrm{p}$.

7. VERSTEEG M.N., 1994. Report of a farmer participatory trip to G hana to obtain knowledge of how and to what extend Mucuna is eaten in Ghanaian households. Benin, IN RA, 6 p.

Accepté le 01.02.2001

\section{Resumen}

O nana J., Yonkeu S. Un enfoque de los costos de producción de semillas forrajeras en la zona sudano sahariana de Camerún

Se evaluaron los costos de producción de semillas de siete leguminosas forrajeras en la zona sudano sahariana de Camerún. Fueron inferiores a $1000 \mathrm{FCFA} / \mathrm{kg}$ para Calopogonium mucunoides, Cajanus cajan, Desmodium distortum, Mucuna pruriens y Stylosantes hamata (1 FCFA $=0,01 \mathrm{FF}$ ). La producción de semillas de Centrosema pascuorum y de Macroptilium lathyroides, con costos respectivos de $1890 \mathrm{y}$ de $3025 \mathrm{FCFA} / \mathrm{kg}$, no parece adaptada a la popularización en la zona estudiada.

Palabras clave: Leguminosa forrajera - Producción de semillas Region Sudano-Saheliana - Camerún. 